\title{
Evaluación del aprendizaje en materias cumiculares: un estudio de caso
}

\author{
ROSA MARÍA MARTÍNEZ RIDER \\ Escuela de Bibliotecología e Información \\ de la Universidad Autónoma de San Luis Potosí \\ E-mail: rrider@uaslp.mx
}

\begin{abstract}
RESUMEN
Se presentan los resultados de la evaluación formativa del aprendizaje en la materia Teońa del Conocimiento Bibliológico-informativo que forma parte del Plan de Estudios 98 de la Escuela de Bibliotecología e Información (EBI) dela Universidad Autónoma de San Luis Potosí (UASLP), la cual pretende desarrollar en los estudiantes un sistema de conocimientos, habilidades y actitudes que le permitan enfrentar los nuevos retos y desafíos, y equilibrar la formación humanística con la tecnológica.

Palabras clave: Evaluación de Programas de Estudio, Universidad Autónoma de San Luis Potosí, Escuela de Bibliotecología e Información.

\section{LEARNING EVALUATION OF THE SUBJECT "THEORY OF KNOWLEDGE IN LIBRARY AND INFORMATION SCIENCE" IN THE CURRICULUM OF THE SCHOOL OF LIBRARY AND INFORMATION SCIEnCE of the Autonomous University of SAN Luis Potosi ROSA MARÍa MARTÍNEZ-RIDER}

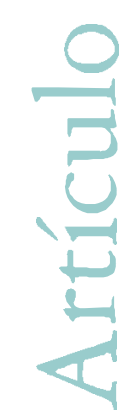

Trabajo recibido el 18 de junio de 2001 $*$ Trabajo aceptado el 4 de julio de 2001

\begin{abstract}
ABST RACT
Results are presented of the training and learning evaluation of the course on the theory of knowledge in library and information science given as part of the study program 98 of the School of Library and Information Science (EBI) of the Autonomous University of San Luis Potosi. This course attempts to develop a system of knowledge, skills and attitudes in the students that allow them to confront new goals and challenges, balancing training in the humanities with technology.

Key words: Study Programmes Evaluation, Universidad Autónoma de San Luis Potosí, Escuela de Bibliotecología e Información.
\end{abstract}

\section{LA EVALUACIÓN CURRICULAR}

도 curriculum desempeña un papel muy importante en cualquier institución educativa no es sencillo definirlo por ser un término polisémico y polimórfico. No obstante su ubicación epistemológica y teórica le da 0 un sentido estrecho y fragmentado, o uno amplio y totalizador, aunque en cualquiera de sus concepciones se ve articulado por factores históricos, filosóficos, psicológicos, sociales, económicos, políticos y culturales. Representa entonces el proyecto de hombre que se pretende formar para responder a las expectativas de la sociedad actual. 
El alumno obtiene aprendizaje formal en el aula dentro de un programa escolarizado, sin embargo también aprende del curriculum oculto mediante la adaptación a las normas, la relación con profesores y con sus compañeros, la legitimación de ideas o creencias, el liderazgo, etcétera. En este contexto, Gimeno Sacristán distingue ánoámbitoscon base en los cuales se puede analizar el curriculum, pero destaca que para "...quienes lo entienden como un campo práctico... supone la posibilidad de: 1. Analizar los procesos instructivos y la realidad desde la práctica desde una perspectiva que les dota de contenido. 2. Estudiarlo como territorio de intersección de prácticas diversas que no solo se refiere a los procesos de tipo pedagógico. 3. Vertebrar el discurso sobre la interacción entre la teoría educativa." ${ }^{1}$

Existen varios elementos en el curriculum:

* La línea de orientación filosófica y pedagógica

* El perfil de ingreso y de egreso

* El mapa curricular

* Las asignaturas

*a vinculación (servicio social, prácticas profesionales, etcétera)

El perfil docente

* Los procesos académico-administrativos

*a investigación educativa y disciplinar, etcétera.

Todos estos elementos se concretan en la formación de los estudiantes mediante la apropiación de los contenidos extraídos de la realidad social.

Siendo la evaluación una parte fundamental para determinar el grado de articulación entre el curriculum y la realidad social, una propuesta para la valoración curricular, plantea interrogantes que abarcan las actividades de aprendizaje y consideran el contexto institucional y social haciendo énfasis en la articulación de los diferentes componentes del curriculum.

Para este trabajo tienen especial relevancia los contenidos y las experiencias de aprendizaje fundamentadas epistemológica, teórica e ideológicamente que buscan entender por qué se aprende y se valora de una manera y no de otra.

En lo relativo al plan de estudios se consideran el mapa curricular y los programas de asignaturas o unidades didácticas que guían los logros que se van a alcanzar en un ciclo escolar, y que deben caracterizarse por la secuencia, integración y continuidad de un sistema de conocimientos, habilidades y actitudes. Tales unidades especifican dentro del proyecto formal o del "deber ser" los objetivos, por alcanzar; los contenidos que se van a cubrir; las actividades de aprendizaje que se van a realizar; la metodología que se seguirá a lo largo del curso; las formas de valoración, y la bibliografía que determina el tipo de conocimiento e ideología que se distribuye socialmente a través de los egresados de un curriculum escolar.

1 Gimeno Sacristán, J., p. 15. 
Tanto la evaluación institucional como la curriculary la del aprendizaje son necesarias especialmente porque "La sociedad que tenemos es absolutamente competitivay el examen contribuye al ejercicio de la competición frente los compañeros," ${ }^{2}$ aunque existen otros instrumentos que se utilizan para valorar, que señalan cuales son las fortalezas y debilidades en las actividades o tareas que se desempeñan, y para descubrir aciertos, problemas y fallas en este proceso, el cual adopta rasgos diferentes según el escenario en el que esté inmerso y determina dos dimensiones que son dialécticas: la cantidad y la calidad.

Por lo general, los métodos experimentales y cuantitativos se utilizan con validez y fiabilidad para valorar productos ya acabados, con el propósito de constatar lo conseguido y tomar medidas oportunas a mediano o largo plazo. Los métodos enmarcados en los modelos cualitativos son idóneos para evaluar procesos y mediante los datos que se recogen día a día con las técnicas adecuadas, mejoradas durante su realización de forma permanente. Por eso parecen los más apropiados para valorar la enseñanza y el aprendizaje que tiene lugar en las aulas. ${ }^{3}$

D os de esos métodos son la observación en el salón de clases y el empleo de cuestionarios. Un profesor puede tener apreciaciones sobre cómo imparte cátedra y cuál es el aprendizaje de los alumnos, pero al contrastar esto con la visión de los estudiantes puede llevarse muchas sorpresas. Los resultados de una evaluación permanente permiten la redefinición y reestructuración constante de las tareas educativas mediante realimentación.

\section{El CURRICUlum de la Escuela de Bibliotecología E INFORMACIÓN DE LA UNIVERSIDAD AUTÓNOMA DE SAN LUIS РоTOSí (UASLP)}

La evaluación curricular de las unidades didácticas en el Plan de Estudios 90 de la EBI, que detalla el objetivo general, el contenido, la metodología, la evaluación y la bibliografía, encontró que los estudiantes tenían una visión fragmentada del mapa curricular y de las asignaturas, y que aun cuando algunas materias pertenecían a la misma área académica se percibían como aisladas. Las causas de esto fueron que:

1. no se justificó la importancia de la asignatura dentro de la totalidad reticular.

2. no se explicó la relevancia de la materia en el desempeño laboral.

Esto hizo que algunas materias fueran etiquetadas como "de relleno" y carentes de significado, en tanto otras fueron identificadas como asignaturas vacías que no respondían ni se correspondían con otras. Asimismo se percibieron problemas de

2 Casanova, Ma. Antonia, p. 9.

3 Ilid p. 17. 
secuencia, se impartían primero las asignaturas de contenido más especializado y después las de contenido general (por ejemplo Consulta y Servicios de información especializada primero y después Servicios al público; o Conjuntos y probabilidades después de Computación). Esto se sumaba a las incongruencias de la Tabla de Seriación e Incompatibilidades y a temas duplicados en las unidades didácticas, que se rediseñaron después de hacer la evaluación curricular para darle congruencia al Plan de estudios y dotar de significado a todas las materias y darles un sentido profesional.

Se diseñó un nuevo formato para hacer la presentación del programa general del curso, el cual abarca:

1. Datos administrativos: (clave de la materia, números de horas teóricas y prácticas, eje curricular al que pertenece la materia, seriación, etcétera).

2. Objetivos generales: descripción de los logros que se pretenden alcanzar al cursar la materia.

3. Justificación: argumentos académicos sobre la necesidad e importancia de cursar la materia dentro del plan de estudios, y fundamentación basada en la relevancia social de la asignatura como parte del ejercicio de la práctica profesional.

4. Contenido: identifica las unidades que conforman el curso haciendo una breve descripción de su contenido e indicando el producto esperado por cada unidad.

5. Requisitos para cursar la materia en conocimientos, habilidades y actitudes.

6. Metodología: organización del curso especificando las formas de participación del alumno en la clase.

7. Evaluación: detalla los rubros que serán considerados en la valoración final del curso, y especifica requisitos para los exámenes extraordinarios, a título de suficiencia y de regularización.

8. Bibliografía del curso: elabora fichas correspondientes al material impreso y electrónico.

La línea de orientación filosófica y pedagógica en el nuevo plan de estudios es sociocultural. La retícula consta de 56 materias obligatorias divididas en cuatro ejes curriculares: social, humanístico, metodológico y disciplinar. Las características generales de egreso implican:

1. Que se maneja y aplica el conocimiento adquirido en las materias que conforman los diferentes ejes.

2. Que se pueden poner en práctica las habilidades de análisis, síntesis, comparación, clasificación, identificación, creatividad, innovación, razonamiento, comprensión, manejo y aplicación de técnicas, así como las relacionadas con la 
tecnología, planificación, evaluación, comunicación, promoción, distribución, interpretación, explicación, comprensión, iniciativa, decisión y trabajo en equipo.

3. Q ue se asumen actitudes y valores de autoestima, honestidad, ética, discreción, indagación, tolerancia, respeto, participación, interés, superación, desarrollo personal y profesional.

4. Q ue hay un interés por la educación continua.

Las características específicas que se relacionan con el tema de este trabajo son:

1. Identificar las distintas corrientes del pensamiento humano que han influido el conocimiento en el campo bibliotecológico.

2. Identificar a la bibliotecología como una ciencia social y relacionada con el resto de las disciplinas que integran el sistema de conocimientos científicos bibliológico-informativo.

3. Contextualizar la práctica bibliotecológica dentro de las unidades de información y relacionarla con la sociedad.

4. Analizar los fundamentos epistemológicos, teóricos, metodológicos y técnicos de la bibliotecología para proponer soluciones a problemas teóricos 0 prácticos de esta disciplina.

\section{ESTUDIO DE CASO: EVALUACIÓN DEL APRENDIZAJE EN LA MATERIA "TEORÍA DEL CONOCIMIENTO BIBLIOLÓGICO-INFORMATIVO"}

\section{Fuente y metodología empleada}

Esta materia pertenece al Eje Curricular Disciplinar de Fundamentos y está seriada con Introducción a la Bibliotecología, Introducción a la Filosofía y Archivología. El contenido de la asignatura fue diseñado por el maestro Salvador G orbea Portal y la maestra Rosa María Martínez Rider y se representa en un mapa conceptual que se define como: “...un recurso esquemático para representar un conjunto de significados conceptuales incluidos en una estructura de preposiciones" , el cual se propone concentrar las ideas principales y no dispersar la atención sobre puntos que pertenecen a otros programas y no duplicar temas.

El objetivo general pretende ubicar los planteamientos epistemológicos, teóricos, metodológicos y técnicos de la bibliotecología, archivología, bibliografología y ciencia de la información. Tras un exhaustivo análisis, la Comisión Curricular de la EBI decidió partir de la propuesta de Emilio Setién y Salvador G orbea ${ }^{5}$ relacionada con el Sistema de Conocimientos Científicos Bibliológico- Informativo.

4 Ontoria, A.,_ \&. al., pp. 31-5. Giroux, H., p. 280.

5 Setién, E. y S. Gorbea, p. 21. 
El énfasis está centrado en la bibliotecología principalmente en dos aspectos:

1. el problema de la disciplina como ciencia,

2. la problemática social en materia de información.

La asignatura se localiza en el tercer semestre y presenta los fundamentos para ejercer la profesión al tomar una postura ante el conocimiento y los valores y mantener un enfoque crítico-constructivo. Las características generales de la asignatura son: 


\section{Mapa conceptual}

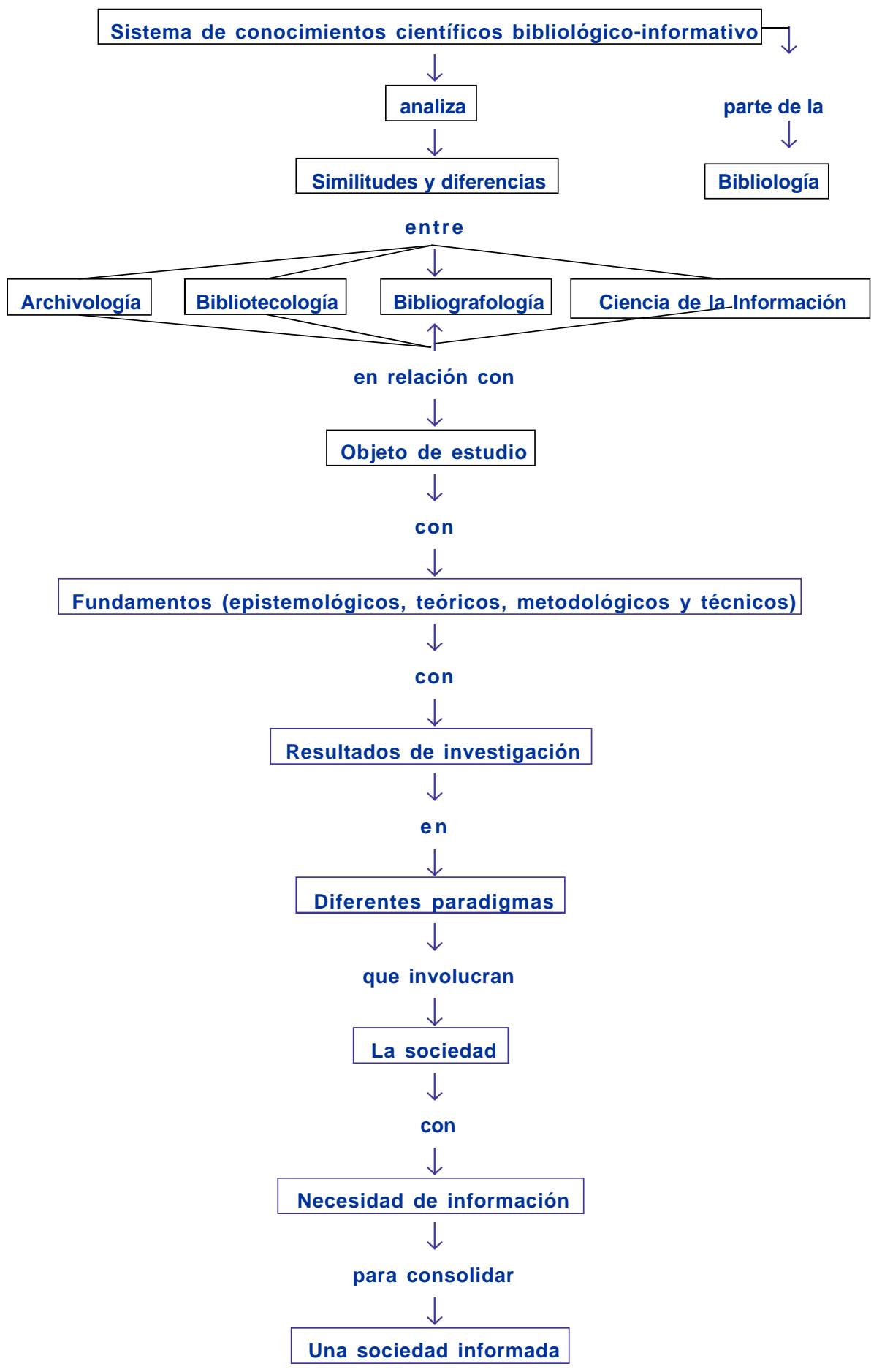


La estructura temática de la asignatura es la siguiente:

\section{Repaso de filosofía general Introdurción \\ * Paradigmas en ciencias sociales \\ * Sociedad: neoliberalismo y humanismo \\ * Información y sociedad}

\section{La arailaciónsoial delosdbumentos Moimientosconvegentes}

Se identifican los movimientos en relación a la creación, recopilación, procesamiento analítico-sintético, almacenamiento, búsqueda, difusión, uso de la información y los sistemas que incorporan nuevos conocimientos. Se analiza la dinámica de la información como apoyo a los sectores sociales en diferentes contextos.

2. El sistemadeconoimientoscientíicosbiblidógicoinformativo

Se analizan las relaciones entre las disciplinas rectoras y las bibliológico- informativas.

3. Labiblidóa

Se analiza su definición identificando su ámbito y contexto.

\section{Labiblidtecoloǵa}

Se analizan los paradigmas de investigación en la disciplina, los objetos de estudio propuestos, los fundamentos epistemológicos, teóricos, metodológicos y técnicos, la bibliotecología como ciencia y la bibliotecología frente a la problemática social.

5. La ardhivdoǵayla adtividadardhiv́stica

Se analiza su objeto de estudio, paradigmas, cosmovisiones y las metodologías cualitativa y cuantitativa.

6. Labibliografotoǵayla adividadbibliogáfica

Se analiza su objeto de estudio, paradigmas, cosmovisiones y metodologías cualitativa y cuantitativa.

7. Lacienia delainfomaciónylaadividaddientíficoinformativa

Se analiza su objeto de estudio, paradigmas, cosmovisiones y las metodologías cualitativa y cuantitativa.

Además de la seriación en el plano del conocimiento los requisitos para cursar la materia de Teoría del conocimiento bibliográfico-informativo. son:

* Apertura para tratar temas filosóficos.

* Razonamiento para fundamentar y argumentar posiciones en la bibliotecología.

* Tolerancia y respeto para escuchar y dialogar aceptando diferentes puntos de vista.

Seguridad para exponer y defender argumentos.

* Identidad que implique una clara postura bibliotecológica. 
El grupo al que se impartió la asignatura se integró con 23 alumnos: 19 mujeres y 4 varones.

El sistema de conocimientos esperados al término del curso está representado en la estructura temática de la asignatura antes presentada y a partir de cada uno de los temas que se indican en la misma.

Las habilidades pretendidas son: análisis, síntesis, razonamiento, argumentación, comparación, identificación, iniciativa, decisión, integración, diferenciación, interpretación, comprensión, explicación, creatividad, asertividad, negociación, evaluación y trabajo en equipo.

Las actitudes que se esperan son: pluralidad, apertura, tolerancia, respeto, seguridad, disponibilidad, cooperación, interés, sensibilidad y paciencia.

Por sus características y contenido esta asignatura exige la crítica como actitud epistemológica; es decir que se puedan encontrar y proponer soluciones a las contradicciones, considerando el contexto y la tricotomía teoría-práctica-realidad, así el estudiante tendrá la oportunidad de aprender el discurso de la asociación pública y de la responsabilidad cívica, y también podría incorporar el análisis desde varios paradigmas no sólo el científico pues “...la reconstrucción ideológica de la realidad mantiene en términos de significación social, los contenidos culturales que el individuo debe internalizar." 6 Fundamentalmente esta asignatura incorpora las bases filosóficas a la bibliotecología y analiza las diferencias que tiene con la archivología y la bibliografología.

Es prioritario que el estudiante maneje una visión holística de la sociedad y que ejercite constantemente el hábito de lectura pues el "...sujeto-lector se transforma, gracias a la lectura, en un lector creador que manifiesta la experiencia lograda como experiencia estética, creando afinidades lectivas en las cuales se habla de gustos, preferencias e intereses, muchos de los cuales se encaminan hacia la concepción del horizonte de expectativas estrechando la relación entre autor-obra-lector." 7

Esto permite que el alumno acceda a una gran variedad de información impresa 0 electrónica; diferencie una gama de posturas, argumentos, razones, opiniones, ideas, creencias, certezas, verdades, posibilidades, probabilidades; aclare dudas; corrija errores y, lo más importante, que defina en su nivel intelectual y espiritual la posición adoptará ante la profesión y la vida, así como los valores que lo regirán como sujeto social y le permitirán alcanzar el objetivo de ser crítico en función de lo que analiza y propone.

El diálogo juega aquí un papel de primer orden para el intercambio de argumentos basándose en el respeto a la forma de pensamiento de otros, por lo tanto no equivale a una simple plática sino a conocer, compartir y construir un conocimiento

6 Muñoz Rosales, V., p. 107.

7 Arizmendi Domínguez, M. A., p. 127. 
conjunto de la bibliotecología y las otras disciplinas que entregan el sistema de conocimientos bibliológico-informativo.

\section{Evaluación del aprendizaje. Análisis de los resultados}

La dosificación es una noción que suele descuidarse no obstante ser el componente que equilibra la dicotomía amplitud-profundidad del conocimiento. "La gradación correcta desde el diseño curricular oficial hasta la unidad didáctica, es la única garantía de que el alumnado está alcanzando los objetivos generales que se proponen para su educación y el aval de que se está ofreciendo desde el ámbito institucional, la igualdad de oportunidades y la enseñanza de calidad que es perceptiva legalmente para todos los ciudadanos." 8 Para determinar si se están logrando los objetivos del aprendizaje que proponen los contenidos programáticos de las asignaturas, el profesor aplica instrumentos que van desde el examen hasta el ensayo 0 reporte de investigación. Sin embargo la administración académica de las instituciones escolares puede también generar otras herramientas de apoyo que proporcionen una guía para que la interpretación de la valoración sea más justa. Cabe aclarar que la finalidad radica únicamente en mejorar las tareas y actividades institucionales, así como el aprendizaje de los alumnos.

La evaluación formativa representa la guía para facilitar la construcción del conocimiento y el desarrollo de habilidades y actitudes. D entro de la teoría pedagógica del constructivismo se plantean dos cuestiones fundamentales:

1. El respeto a las estructuras mentales del alumno mediante la congruencia entre los aprendizajes previos, simultáneos y subsecuentes.

2. La promoción de un aprendizaje significativo que le dé sentido a la vida cotidiana y profesional de los sujetos.

De este modo se intenta dar cuenta de "Los progresos, dificultades, bloqueos, etc. que jalonan el proceso de aprendizaje" y se realiza mediante la observación sistemática y pautada. Con estos resultados existe la posibilidad de modificar las unidades didácticas, los planes de estudio y las curricula. En relación con los contenidos programáticos, se determina:

1. qué aprendió realmente el alumno y qué no aprendió;

2. qué temas proporcionaron un aprendizaje significativo;

3. cuáles fueron los avances más importantes y los obstáculos más difíciles en el aprendizaje;

4. qué elementos fueron importantes para la planificación y toma de decisiones en relación con el contenido o experiencias de aprendizaje del programa de la materia; 
5. cómo se modificó el curriculum en el área, eje o módulo que le corresponda a la asignatura en cuestión;

6. y por tanto una de las características de la evaluación formativa es que no se asigna ningún valor numérico.

La unidad didáctica representa el plan de trabajo anual, semestral, cuatrimestral, etcétera, e incluye la información básica para el desarrollo del curso, pero no debe considerarse como algo dado y acabado sino como la base para reconstruir el conocimiento pues no debe confundirse una educación en libertad orientada hacia una postura epistemológica, con otra de tipo tradicional o libertina, únicamente porque el contenido programático ya está diseñado. Tampoco debe confundirse con la anarquía porque debe partir de una perspectiva filosófica y pedagógica que le permita al alumno apropiarse de la realidad y responder a sus expectativas.

Los procesos de valoración durante el curso se basaron en:

1. la aplicación de un examen diagnóstico;

2. la observación que utiliza fichas de registro;

3. la elaboración de resúmenes, la presentación de investigaciones y la redacción de ensayos académicos sobre los temas del curso, $\mathrm{y}$

4. la aplicación de un cuestionario denominado "Evaluación del proceso enseñanza-aprendizaje" (anexo).

En relación con la aplicación del examen diagnóstico sobre los conocimientos previos adquiridos en Introducción a la Filosofía, los resultados indicaron que los alumnos no definieron con exactitud los conceptos de "epistemología", "materialismo dialéctico" y "materialismo histónico", y que era necesario reforzar algunos planteamientos de la filosofía latinoamericana. Se recomendó al profesor de esta materia que pusiera atención en estas lagunas del conocimiento para su próximo curso.

En cuanto a la observación, sin pretender que ésta tuviera corte etnográfico en sentido estricto, sólo se registraron datos importantes y los puntos de atención para tratar de mejorar el aprendizaje. Se presenta a continuación un cuadro con las dificultades que se encontraron y las estrategias que se utilizaron para facilitar el proceso en el aula. Se hace hincapié en que los estudiantes contaban con conocimientos previos en filosofía y que los alumnos se mostraron muy interesados en los temas sociales. 


\begin{tabular}{|c|c|c|}
\hline & DIFICULTADES & ESTRATEGIAS \\
\hline CONOCIMIENTO & $\begin{array}{l}\text { - Para ubicar conceptos con base } \\
\text { en el paradigma científico } \\
\text { - Para manejar algunos } \\
\text { conceptos epistemológicos } \\
\text { - Para practicar la hermenéutica }\end{array}$ & $\begin{array}{l}\text { - Utilizar lenguaje claro, muchos } \\
\text { ejemplos y analogías } \\
\text { - Ejercer la interpretación }\end{array}$ \\
\hline HABILIDADES & $\begin{array}{l}\text { - Poca participación al principio } \\
\text { - Exposiciones que no utilizan } \\
\text { el tiempo signado (acaban } \\
\text { antes o después) } \\
\text { - Al principio inseguridad en la } \\
\text { exposición }\end{array}$ & $\begin{array}{l}\text { - Utilizar explicación al princi- } \\
\text { pio como introducción } \\
\text { - Utilizar técnicas de discusión } \\
\text { dirigida e interrogatorio }\end{array}$ \\
\hline ACTITUDES & 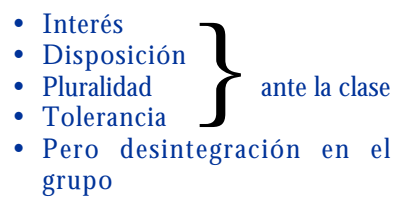 & $\begin{array}{l}\text { - Seleccionar temas de mayor in- } \\
\text { terés bibliotecológico } \\
\text { - Trabajar en equipo }\end{array}$ \\
\hline $\begin{array}{l}\text { RELACIÓN PROFESOR- } \\
\text { ALUMNO }\end{array}$ & $\begin{array}{l}\text { - Cordial } \\
\text { - Participativa (no voluntaria al } \\
\text { principio } \\
\text { - Resistencia a la lectura; } \\
\text { preferían la técnica expositiva }\end{array}$ & $\begin{array}{l}\text { - Justificación de la importancia } \\
\text { de la lectura para poder discu- } \\
\text { tir como parte de la formación } \\
\text { profesional }\end{array}$ \\
\hline $\begin{array}{l}\text { RELACIÓN ALUMNO- } \\
\text { ALUMNO }\end{array}$ & $\begin{array}{l}\text { - Resistencia al trabajo en } \\
\text { equipo } \\
\text { - Integración de equipos por } \\
\text { amiguismo }\end{array}$ & $\begin{array}{l}\text { - Equipos predeterminados por } \\
\text { el profesor, y posteriormente } \\
\text { integración libre de equipos }\end{array}$ \\
\hline CLIMA & $\begin{array}{l}\text { - D inámico } \\
\text { - Cordial } \\
\text { - Expectante }\end{array}$ & $\begin{array}{l}\text { - Técnicas de trabajo que mantu- } \\
\text { vieron el mismo clima }\end{array}$ \\
\hline
\end{tabular}

En cuanto a la elaboración de resúmenes la valoración se hizo en función de la capacidad de extraer los puntos que representaban la esencia de las lecturas, y en la presentación de investigaciones o la redacción de ensayos académicos se consideró lo siguiente: Fundamentación, ya fuera en autores de una misma perspectiva epistemológica y teórica o en diferentes autores; y para su discusión escrita, las opiniones y propuestas de los estudiantes argumentadas y razonadas; también se tomó en cuenta la presentación física del documento.

Con referencia al cuestionario del anexo: "Evaluación de proceso enseñanzaaprendizaje” éste se aplicó después de cada tema (ocho en total). A continuación se presentan algunos resultados obtenidos al aplicarse en diferentes momentos a lo 
largo del curso: uno después de revisar los contenidos de la Introducción, y otros después de cada unidad restante en orden sucesivo.

\section{Temas revisados en la unidad (mismo resultado en los cuestionarios de las 8 teonías)}

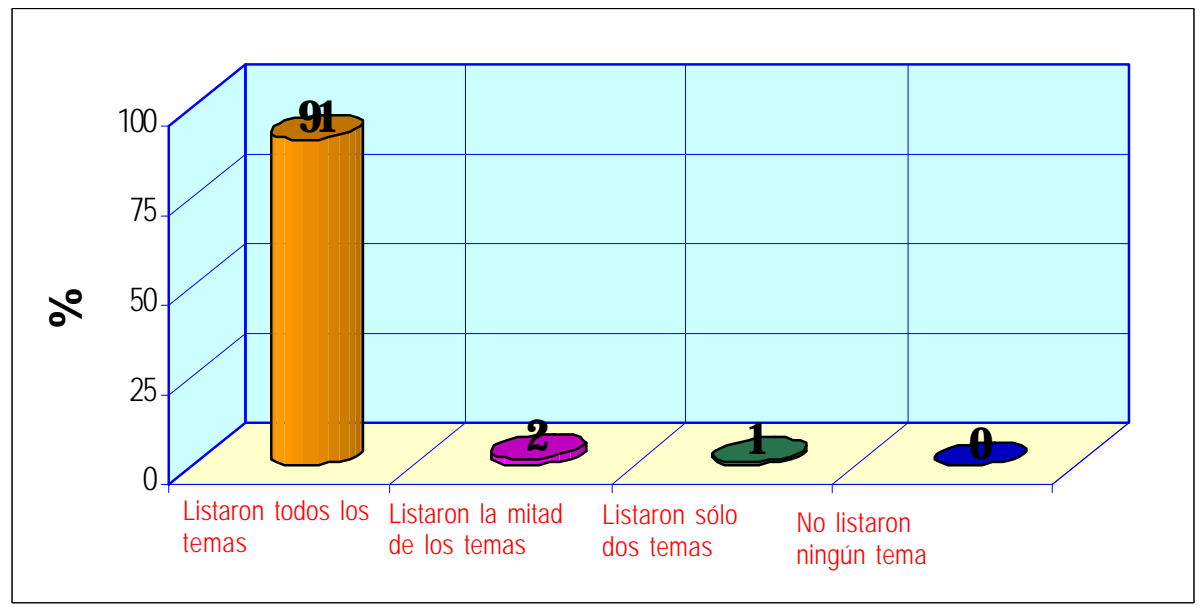

E strategia: Se volvieron a repasar los temas completos a todo el grupo entre los estudiantes del grupo que no listaron todos los temas.

Conceptos que no quedaron claros de los temas (resultados en los cuestionarios de los ocho temas)

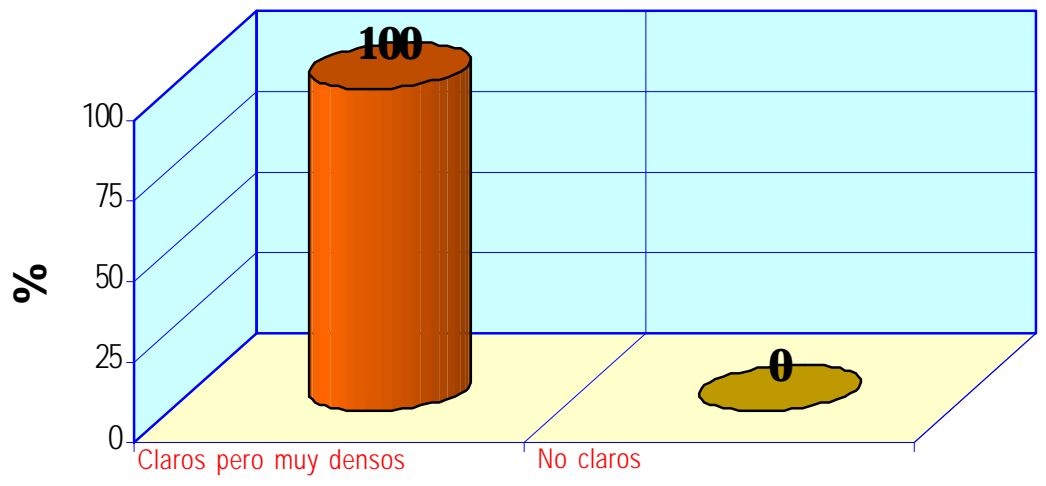

Estrategia: Con la técnica de interrogatorio explicar nuevamente los temas que se consideraron densos. 


\section{Dificultades en el aprendizaje sobre los temas aprendidos (Unidad IV. La Bibliotecología)}

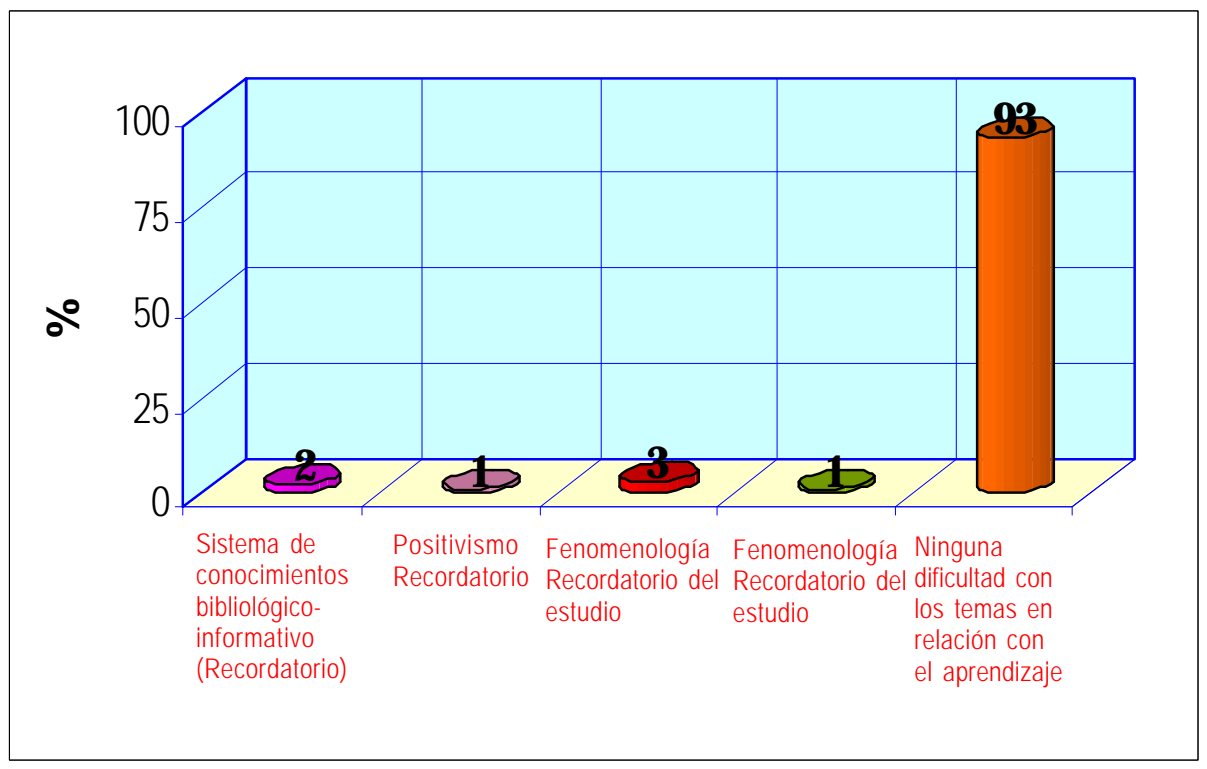

Estrategia: Explicar cuidadosamente los temas que representaron dificultad en el aprendizaje

\section{Temas que se desearían ampliar (Unidad IV. La Bibliotecología)}

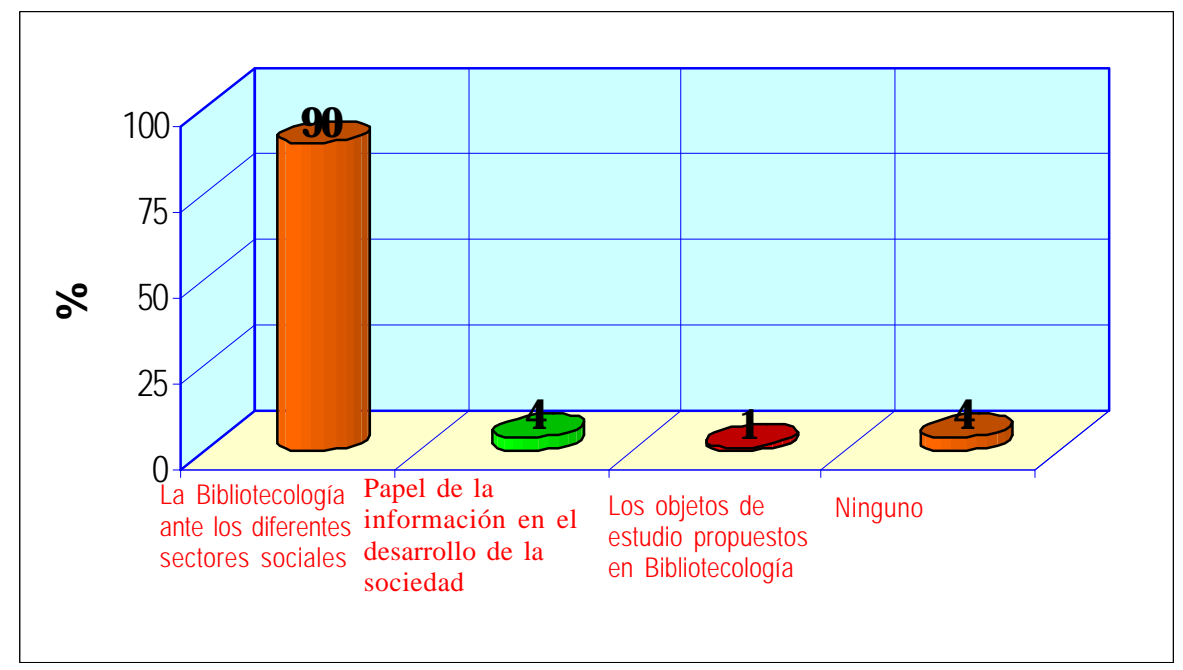

Estrategia: Ampliar el aprendizaje con lecturas, películas y otros medios audiovisuales. 
Sugerencia de los alumnos para aprender mejor la materia (resultado en cuestionarios de los temas Introducción, I, II, III, IV, y V)

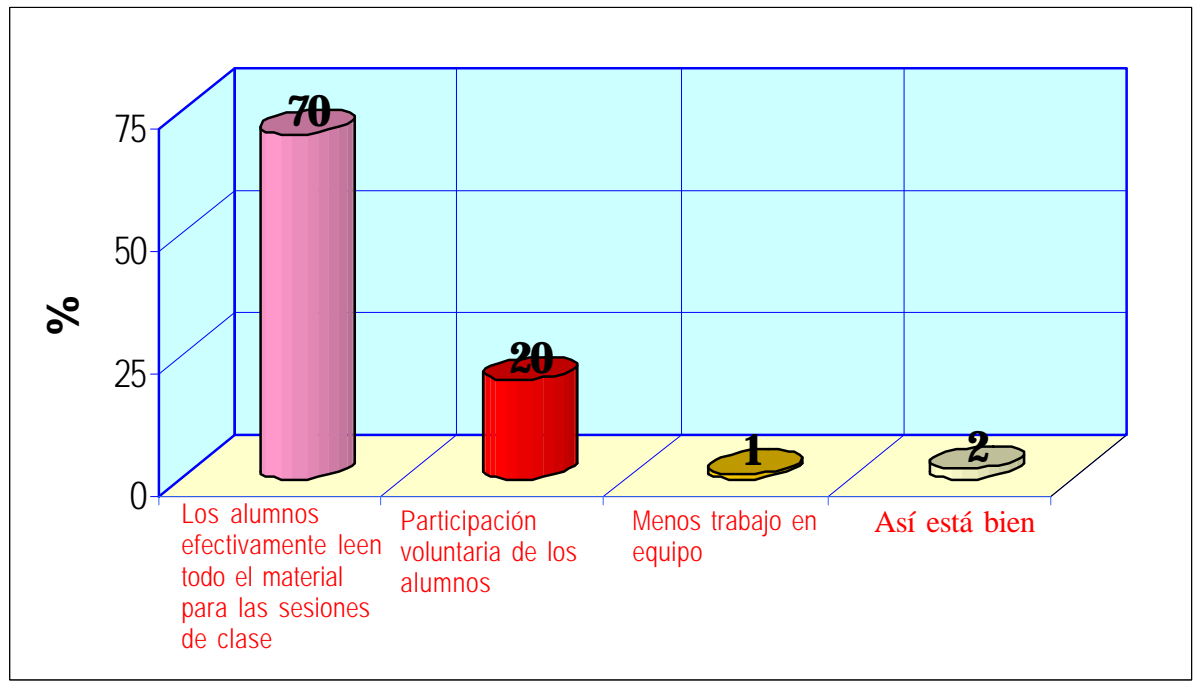

E strategia: Recomendar a los estudiantes que trabajaran en tales debilidades (procurar leer más, mayor participación, etcétera).

\section{Sugerencias sobre como les gustaría a los alumnos} que se impartiera la asignatura (resultado en cuestionarios de los temas Introducción, I, II, III, IV y v)

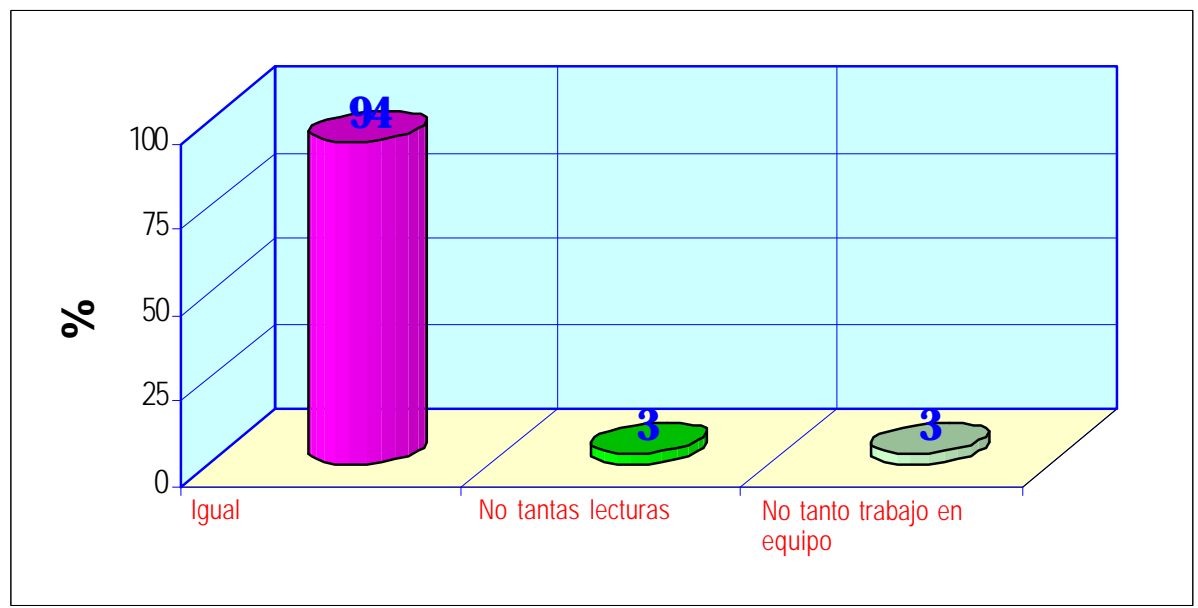

E strategia: Justificar la importancia de la lectura y el trabajo en equipo para el bibliotecólogo en formación. 


\section{Lo que no les gusta a los alumnos de la clase (mismo resultado en los temas Introducción, I y II)}

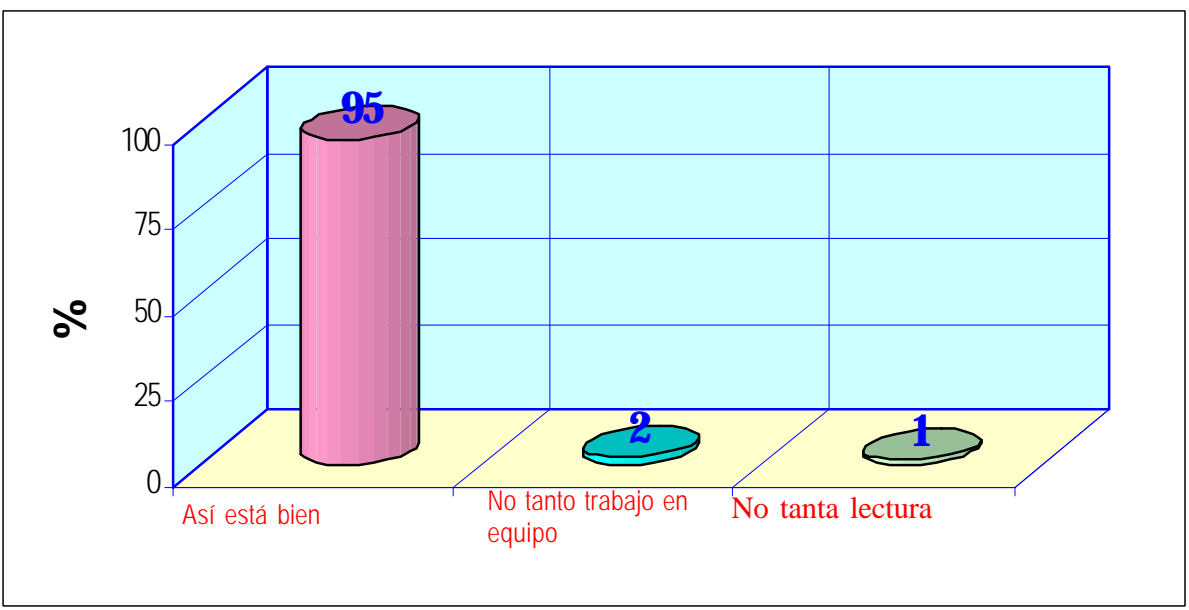

Estrategia: Justificar la importancia de la lectura y el trabajo en equipo para el bibliotecólogo en formación.

\section{Importancia de los temas en el programa de la materia (cuestionario tema IV. La Bibliotecología)}

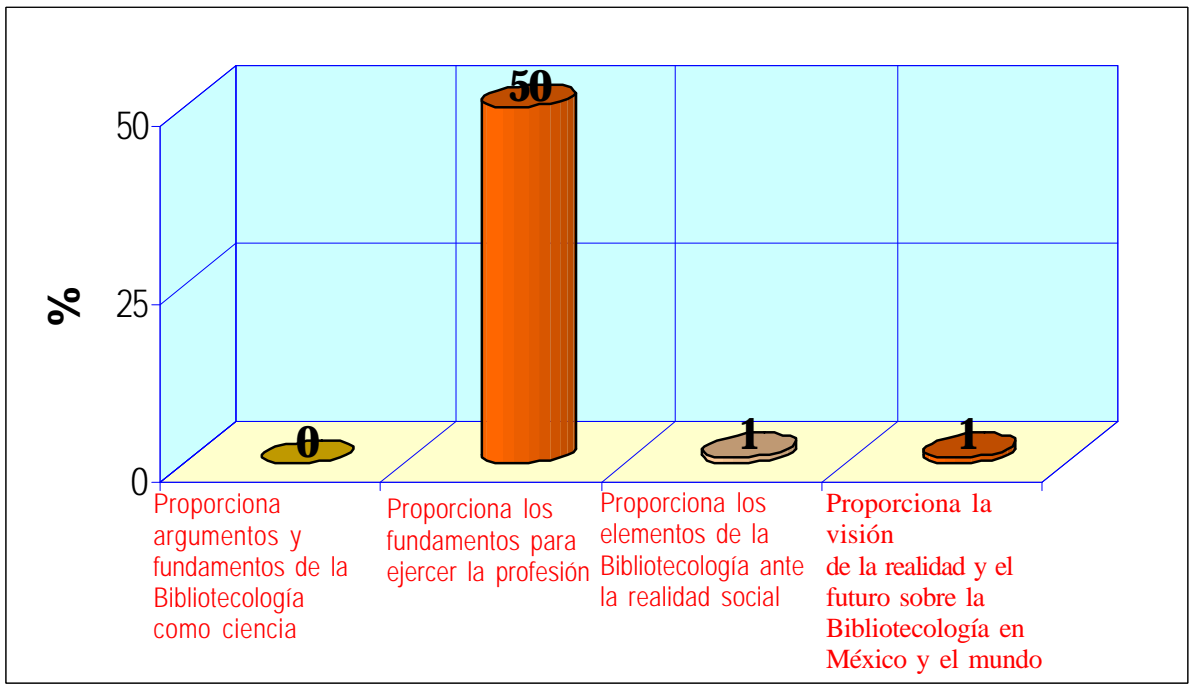

Estrategia: Reafirmar que la importancia radica en integrar las cuatro respuestas que dieron. 
¿Qué otras materias del plan de estudios apoya?

"Teońa del conocimiento bibliotecológico informativo" (mismo resultado tema I y II)

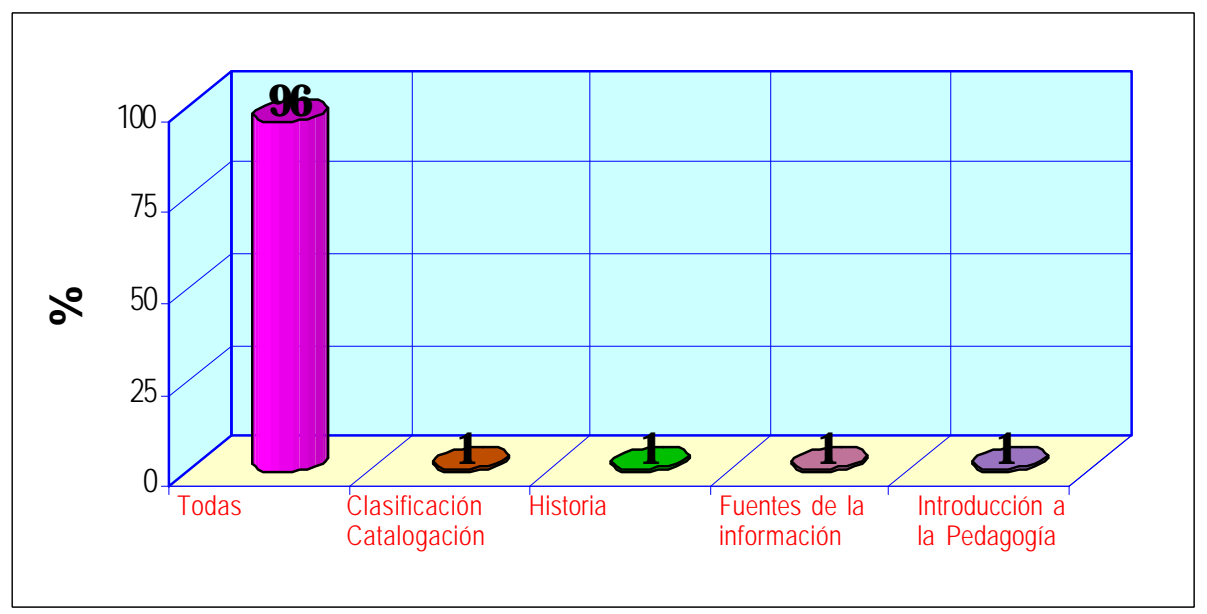

Estrategia: Explicar que en realidad apoya todas las materias del plan de estudios, pues esta asignatura representa el fundamento de la profesión.

\section{Utilidad de esta asignatura en el desempeño profesional (tema IV. La Bibliotecología)}

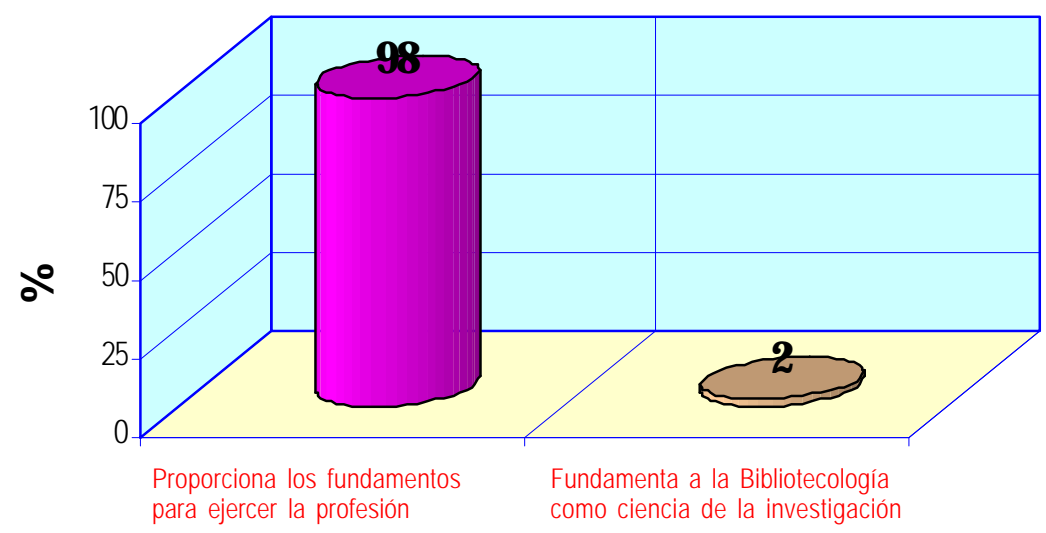

Estrategia: Explicar que las dos respuestas son integrales. 
Los principales problemas que se dieron durante parte del semestre al interior del grupo fueron la resistencia al trabajo en equipo y a la lectura. Se insistió en la importancia de ambos en la formación profesional.

\section{CONSIDERACIONES FINALES}

1. La evaluación del aprendizaje redunda en la calidad del egresado por eso es importante que el profesor se preocupe de que el alumno asimile y reflexione verdaderamente sobre lo enseñado.

2. Existen muchas estrategias - desde la observación hasta la entrevista y el cuestionario para evaluar el aprendizaje- que arrojan datos que permiten corregir el proceso formativo en la escuela.

3. Los resultados de la evaluación formativa deben utilizarse para corregir 0 modificar las partes débiles del proceso de aprendizaje y reforzar las de mayor interés.

\section{BIBLIOGRAFÍA}

ARIZMENDi D omínguez, M. A. “Un horizonte para la lectura; la Teoría de la Recepción”. Coatepee4: 125-129, 1995.

CASANOVA, M. A. Manual deealuaaóneedrativa. Madrid, La Muralla, 1995

Coli, C. Psicdoǵayamialum México, Paidós, 1992

G IMENO Sacristán, J. El amiaulum una rellexión sdrela prádica 21a. ed, Madrid, Morata, 1989.

GIRo ux, H. Laesadaylaludhaporlaciudadanía México, Siglo XXI, 1993.

MuÑoz Rosales, V. "Discurso y práctica educativa”. Filosfíadelaecuracoón: Haáa una peedagogáa paraAméica Latina. México, UNAM, 1993

O NTO Ria, A. e. al. Mapas coneqpurales una ténica para aprender. 5a. ed., Madrid, Narcea, 1995.

PÉREZ Gómez, A. L. "Elaprendizaje escolar: de la didáctica operatoriaa la reconstrucción de la cultura en el aula". Comprendar y transfommar la enseñanza Madrid, Morata, 1992.

PROYECTO de Reestructuracón Cumiailar para la Esada de Biblidtedoǵa e InformacoóndelaUnivesidadAutónna deSanLuisPotoś; PlanInstituciond deDesandlo1997-2007. "O ferta educativa y diseño curricular". Rosa María Martínez Rider (coord.), Salvador G orbea Portal (asesor), San Luis Potosí, Universidad Autónoma de San Luis Potosí, 1998. 
RIDER Martínez, Rosa María y Salvador G orbea Portal. "Integración delas matemáticas y las ciencias bibliotecológicas y de la información: una necesidad de los nuevos planes de estudio de la licenciatura". Investicaaón Bibidtedóǵca (México) 12 (24): 117-132, enero/ junio de 1998.

SETIÉN Q uesada, E milio y Salvador G orbea Portal. "D e la Bibliotecología al Sistema de Conocimientos Científicos Bibliológico-Informativo". InvestigaiónBiblidtedớca (México) 8 (16): 21-25, 1994.

ZARZAR Charur, C. "Conductay aprendizaje: una aproximación teórica". Pefiles educativos (17): 27-42, 1982.

\section{ANEXO I}

\begin{tabular}{|c|c|c|}
\hline \multicolumn{2}{|c|}{ ESCUELA DEBIBLIOTECOLOGর্A EINFORMACIÓN } & EJES \\
\hline Social & Humanístico & Metodológico \\
\hline $\begin{array}{l}\text { - Introducción a la Psicología } \\
\text { - Introducción a la Pedagogía } \\
\text { - Teoría de la Comunicación } \\
\text { - Relaciones Humanas } \\
\text { - Administración General } \\
\text { - Administración de Recursos } \\
\text { - Humanos } \\
\text { - Legislación y Normalización } \\
\text { - Contexto de la Información } \\
\text { en México I, II } \\
\text { - Diseño de Edificios para } \\
\text { Unidades de Información }\end{array}$ & $\begin{array}{l}\text { - Introducción a la Filosofía } \\
\text { - Taller de Lectura y Redac- } \\
\text { ción } \\
\text { - Historia de la Cultura I, II } \\
\text { - Historia de la Ciencia } \\
\text { - Historia del Libro y las Bi- } \\
\text { bliotecas } \\
\text { - Seminario de Historia de } \\
\text { México }\end{array}$ & $\begin{array}{l}\text { - Matemáticas } \\
\text { - Probabilidades y Estadística } \\
\text { - Metodología de la Investiga- } \\
\text { ción } \\
\text { - Seminario de Investigación } \\
\text { Bibliotecológica }\end{array}$ \\
\hline
\end{tabular}

\section{ANEXO II \\ Universidad Autónoma de San Luis Potosí Escuela de Bibliotecología e Información}

\section{Cuestionario \\ Evaluación del proceso enseñanza-aprendizaje}

Por favor contesta este cuestionario con toda honestidad, el beneficiado serás tu mismo, porque me ayudarás a que esta materia sea interesante para ti.

1 .Enumera los temas revisados en la unidad

2. D e estos temas ¿en cuáles se te ha dificultado más el aprendizaje? ¿Por qué?

3. ¿Q ué temas te gustaría ampliar?

4. ¿Q ué sugerencias puedes hacer para aprender la materia?

5. ¿Cómo te gustaría que se diera la clase?

6. ¿Q ué no te gusta de la clase? 
7. ¿Por qué son importantes los temas que se han revisado en esta unidad dentro de la materia que cursas?

8. ¿Crees qué esta materia apoya a otras asignaturas del plan de estudios? ¿Cuáles?

9. ¿Qué utilidad crees que te darán estos temas en tu desempeño como bibliotecólogo , cuando labores en una unidad de información?

10. Anota otros comentarios que quieras hacer en relación a esta materia. 